\title{
Making Nuclear Physics Industrial Engineering Science: Talented Soviet Physicist with Troublesome Character and Career
}

\author{
Nurgaliev Ildus S. \\ United Nations Educational, Scientific and Cultural Organization (UNESCO) Chair in Renewable Energy and Rural Electrification, \\ All-Russian Research Institute for Electrification of Agriculture, Moscow, Russian Federation
}

Email address:

ildus58@mail.ru

\section{To cite this article:}

Nurgaliev Ildus S. Making Nuclear Physics Industrial Engineering Science: Talented Soviet Physicist with Troublesome Character and Career. American Journal of Mechanical and Industrial Engineering. Vol. 2, No. 3, 2017, pp. 144-149. doi: 10.11648/j.ajmie.20170203.15

Received: November 1, 2016; Accepted: January 6, 2017; Published: March 6, 2017

\begin{abstract}
The biography and scientific results of the prominent Soviet-Russian physicist Dmitry Ivanenko are presented. Dramatic career of the talented physicist is described on the historic background of dramatic period of Soviet Union. Professor Ivanenko appears as an figure of Novel level by his talent of Physicist not get recognized by part of colleagues because of his personal troublesome character and historic circumstances. The purpose of the article is to bring attention of the community to necessity of closer collaboration between scientists and state governments in front of the coming global challenges.
\end{abstract}

Keywords: Industrial Engineering, Biography of D. Ivanenko, History of Theoretical Physics, Mechanical Engineering, Cosmological Term, Theory of Gravitation

\section{Introduction}

Every period of science history has its heroes and, sometimes, anti-heroes. Sometimes they change their status in accordance with further scientific development, with relationships with colleagues and with power. The decades after the Russian Revolution, called Great October Socialistic Revolution in the Soviet Union those years, were such kind of period for "Jazz Band": Lev Landau, Geore Gamov, Matvei Bronstein and Dmitri Ivanenko, young talented Physicists in Leningrad in the mid-1920s who were passionately studying cutting edge dynamic theoretical Physics of those years. The name "Jazz Band" means that American Jazz for a short period, before getting banned, symbolized for the quartet freedom, upbeat lifestyle and optimism. All members of the cheerful "band" experienced harsh repressive character of the regime. Landau and Ivanenko were repressed, Gamov defeated, Bronstein was shot. In 1980s once when Dmitry Ivanenko called his group "our band" in our converstion Gennady Sardanashvili reacted "Dmitri Dmitrievich the term "band" is not acceptable for scientific group and sounds very strange". He was right because in Russian context this term sounds as "gang" for those who does not know the English connotation of the Russian word "band". This report has memorial character and refers to the scientific results of the author only in the list of references.

\section{Biography of Dmitri Ivanenko}

Dmitri Dmitrievich Ivanenko (1904-1994) was one of the prominent theoreticians and actively contributed into gravitational and nuclear research as well as to the institutional development of this branches of Physics in the former Soviet Union.

He was born on July 29, 1904 in Poltava (Russian Empire), currently Ukraine Republic, in a family of well educated parents. His father was editor and publisher of the city newspaper, his mother was a school teacher. In 1920 he graduated from high school in Poltava. From 1920 to 1923 he worked as a teacher of physics and mathematics at a school of Poltava, while he studied and graduated from the Poltava Pedagogical Institute and worked part time at the Poltava Astronomical Observatory. He was enrolled in Kharkov University, and at the first course was transferred to the Leningrad State University, from which he graduated in 1927. His attempt to transfer from Kharkov University into Moscow State University was not successful, though, he, much later, became famous as a professor of the Moscow 
State University.

In 1927-1929 he was student of the Leningrad branch of the Physics and Mathematics Institute of the USSR awarded the scholarship named after Steklov, and a research fellow of the same institute. In 1929 he moved to Kharkov, to lead the theoretical department of Ukrainian Physico-Technical Institute.

Dmitri Ivanenko worked at the Kharkov Institute of Physics and Technology in 1929 - 31, being the first Director of its theoretical department; Lev Landau, Nobel Laureate, and student-years friend and later rival, became his successor, 1932 - 37. Paskual Jordan, Victor Wieskopf, Felix Bloch (Nobel laureate in 1952) and Paul Dirac (Nobel Laureate in 1933) visited D. Ivanenko in Kharkov. Ivanenko very often used to mention with well-deserved pride his role in organizing the $1^{\text {st }}$ Soviet theoretical conference (1929) in Kharkov, and founding the journal "Physikalische Zeitschrift der Sowjetunion" first Soviet journal in foreign language (1932). In February 1931, his rank of professor was approved by the Presidium of the Supreme Economic Council of the Ukrainian SSR. He became a head of the Department of Theoretical Physics of the Kharkov Mechanical Engineering (former Technology) Institute of Kharkov University.

Since 1931 again in Leningrad, he became senior researcher at the Leningrad Physico-Technical Institute named after Abraam Ioffe, as well as the head of the seminar on nuclear physics. He served as the editor of the theoretical department of the Leningrad branch of the State technical and theoretical publishing house in 1932-1935. Eight collections of classical works and books of modern physics were published under his editorship the first time in Russian including books of Lui deBroile, Heisenberg, Dirac, Schrödinger, Brilluen, Sommerfield, Eddington. D. Ivanenko was professor, head of the Department of Physics of the Leningrad Pedagogical Institute named after Pokrovsky.

After returning to Leningrad at the Physical-Technical Institute, D. Ivanenko concentrated his interest in nuclear physics. In May 1932, Ivanenko published the proton-neutron model of the atomic nucleus in journal "Nature", and two months later Werner Heisenberg (Nobel laureate in 1932) referred to his work. In August 1932, D. Ivanenko and E. Gapon proposed a pioneer nuclear shell model describing the energy level arrangement of protons and neutrons in the nucleus in terms of energy levels. Later this model was developed by Eugene Paul Wigner, Maria Goeppert-Mayer and J. Hans D. Jensen who shared the 1963 Nobel Prize for their contributions.

Ivanenko's success pushed forward the nuclear physics progress in the former USSR. In 1933 on the initiative of Dmitri Ivanenko and Igor Kurchatov, the $1^{\text {st }}$ Soviet nuclear conference was organized. Paul Dirac, Frédéric Joliot-Curie (Nobel laureate in 1935), Fransis Perrin, Franco Rasetti, Victor Weisskopf et al participated in this Conference.

The realization of Ivanenko's far-reaching plans and hopes was interrupted abruptly in 1935 -- he was arrested in the context of the intensification of the repressions after political leader Sergey Kirov assassination. Presumably, he was accused in participation in anti-soviet activity in connection with defeated George Gamow. Exile to Tomsk followed after Karaganda forced labor camp. D. Ivanenko was a professor at Tomsk and Sverdlovsk Universities until the beginning of the World War II. From 1943 and until the last days of his life, he was closely associated with the Physics Faculty of M. V. Lomonosov Moscow State University.

Dmitri Ivanenko made the fundamental contribution to many areas of nuclear physics, field theory and theory of gravitation. Here are the details in short. In 1928, Ivanenko and Landau developed the theory of fermions as skew-symmetric tensors in contrast with the Dirac spinor model. Their theory, widely known as the Ivanenko -Landau - Kahler theory, is not equivalent to Dirac's one in the presence of a gravitational field, and only one describing fermions in contemporary lattice field theory.

In 1929, Ivanenko and Fock generalized the Dirac equation and described parallel transport of spinors in a curved space-time (the famous Fock - Ivanenko coefficients). Nobel laureate Abdus Salam called it the first gauge field theory.

In 1930, Victor Ambartsumian and Dmitri Ivanenko suggested the hypothesis of creation and annihilation of massive particles which became the corner stone of contemporary quantum field theory.

Dmitri Ivanenko and Igor Tamm (Nobel Laureate in 1958) suggested the first non-phenomenological theory of paired electron-neutrinor nuclear forces in 1934. They made the significant assumption that interaction can be undergone by an exchange of massive particles. Based on their model, Nobel laureate Hideki Yukawa developed his meson theory.

Ivanenko proposed a non-linear generalization of Dirac's equation in 1938. Based on this generalization, W. Heisenberg and he developed the unified nonlinear field theory in 50ths. In 1944, Dmitri Ivanenko and Isaak Pomeranchuk predicted the phenomenon of synchrotron radiation from relativistic electrons in a betatron. This radiation was soon discovered experimentally by American experimenters D. Bluitt (1946) and H. Pollock (1947). Synchrotron radiation possesses a number of very specific properties which provide its wide applications. In particular, neutron stars also are sources of this type of radiation. Classical theory of synchrotron radiation was developed by Dmitri Ivanenko in collaboration with Arseny Sokolov in 1948, and independently by Julian Schwinger (Nobel Laureate, 1965). For their work on synchrotron radiation, D. Ivanenko, A. Sokolov and I. Pomeranchuk were awarded the Stalin Prize in 1950.

Two of D. Ivanenko's and A. Sokolov's monographs "Classical Field Theory" and "Quantum Field Theory" were published at the beginning of the 50s. "Classical field theory" was the first contemporary book on field theory where, for instance, the technique of generalized functions was applied. Nobel laureate Ilya Prigogine referred to it as text-book he studied. In 1956, D. Ivanenko developed the theory of hypernuclei discovered by Marian Danysz and Jerzy Pniewski in 1952. 
At the beginning of the 1960's, D. Ivanenko concentrated in intensive scientific and organizational work on the development and coordination of gravitation research in the USSR. In 1961, under his initiative the $1^{\text {st }}$ Soviet gravitation conference was organized. D. Ivanenko was the founding father of Soviet Gravitation Commission, which existed until the 1980's. He served as an active member of the International gravitation Committee since its founding in 1959. In the $70-80 \mathrm{~s}$, D. Ivanenko was concentrated on gravitation theory. He and his pupils developed different generalizations of Einstein's General Relativity, including gravity with torsion and gauge gravitation theory. In 1985, D. Ivanenko and his collaborators published two monographs "Gravitation" and "Gauge Gravitation Theory".

Theoretical physics in the USSR has been enormously influenced by the seminar on theoretical physics organized by D. D. Ivanenko in 1944 that has continued to meet for 50 years under his guidance at the Physics Faculty of Moscow State University and became one of most famous in the USSR. The distinguishing feature of Ivanenko's seminar was grasp of the cutting edge problems of theoretical physics and its broad inter-disciplinary discussion, for example, gravitation theory and elementary particle physics. The most prominent physicists in the world participated in the seminar: Niels and Aage Bohr, Paul Dirac, Hideki Yukawa, Julian Schwinger, Abdus Salam, Ilya Prigogine, Samuel Ting, Paskual Jordan, Tullio Regge, John Wheeler, Roger Penrose et al.

The scientific style of Dmitri Ivanenko was characterized by great interest in a broad spectrum of frontiers' ideas in science where these ideas were based on strong fundamental mathematical methods or experiment. Seven Nobel Laureates: P. A. M. Dirac, H. Yukawa, N. Bohr, I. Prigogine, S. Ting, M. Gell-Mann, G. 't Hooft wrote their famous inscriptions with a chalk on the walls of Ivanenko's office in Moscow State University.

Following to the suggestion by professor Tilman Sauer I want to add some new facts discovered recently when I was preparing reports and publication devoted to 100-year jubilee of Academician Yakov Borisovich Zeldovich. Some historically valuable facts about Landau and Ivanenko from their student years were reported by Academician Isaak Markovich Khalatnikov remembering stories by Landau.

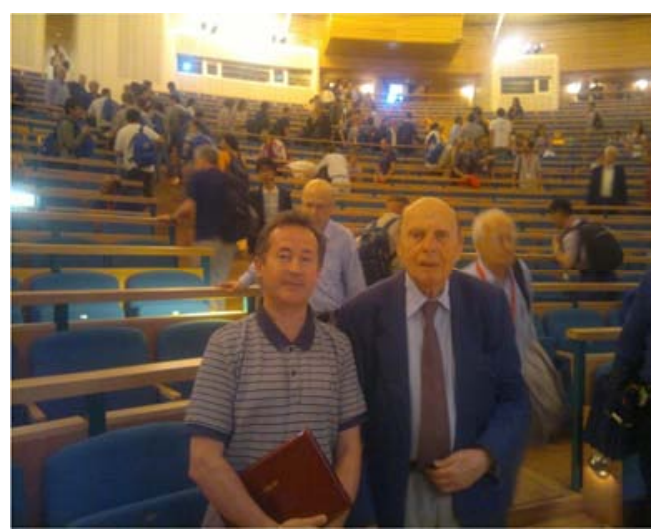

Figure 1. Academician Isaak Markovich Khalatnikov and the author on Marcel Grossman Meeting in 2012, Stockholm.
There was a study group in Leningrad State University at the time which consisted of very talented theoretical physicists: Lev Landau, George Gamow, Matvey Bronstein, Dmitri Ivanenko. Vladimir Fok was the leader of the team. They used to use in this group to address each other by short nicknames instead of names. Landau became Dau, Bronstein was called Abbat, Ivanenko got nickname Dimus, and Gamow became Johnny. The apartment of a young poetess Zhenya Kannegisser served their sort of at-home club. Zhenya was a cousin of Leonid Kannegisser who shot the Chairman of the Cheka (secret police, predecessor of NKVD), M. S. Uritsky (influential soviet politician of Stalin period). The entire company used to gather at place to have good time; and the evenings were full of jokes, music, intellectual excitement and reading poetry. As we hear from memoirs, this behavior was not typically the "soviet type", contrary, might accepted by some solid guardians of orders as too frivolous. From the distance of so many years it may be concluded that multiple conflicts between power and intellectuals started from the sort of minor "cultural mutual misperceptions" between them growing later up to dramatic conflicts. This happen, in different extents, to the all members of the group "Jazz Band", as they called themselves.

At some moment a young British physicist Rudi Peierls was also introduced there, he felt in romance with Zhenya and soon they get married. Subsequently, when Rudi Peierls became Sir Rudi, she became Lady Peierls. R. Peierls was the Head of Theoretical Department in the Los Alamos Laboratory. As Academician Khalatnikov (figure 1) pointed out [17], one of his closest coworkers was to be later famous spy Emil Julius Klaus Fuchs (29 December 1911 - 28 January 1988). He convicted of supplying information from the American, British, and Canadian Manhattan Project to the Soviet Union during and shortly after the Second World War. While at the Los Alamos National Laboratory, Fuchs was responsible for many significant theoretical calculations relating to the first nuclear weapons, and later, early models of the hydrogen bomb.

In 1968, when Isaak Markovich Khalatnikov visited England, Lady Peierls told him that she and her husband, Sir Rudi, were visiting Fuchs on Sundays, in a British prison. One has to note that Fuchs was a staunch anti-Fascist and absolutely disinterestedly in egoistical terms, rather, based on his political anti-Fascistic principles, supplied information to the Soviet Union.

This last fragment, written after consulting with professor Tilman Sauer, brings us to paramount interesting topic of the interaction between physicists and state governments over security issues. In my earlier works as well as latest publications, commemorated Yakov Zeldovich, specifically opposing attitudes of Ya. Zeldovich and A. Sakharov (Nobel Peace Prize laureate) $[5,19]$ over principles of serving to the country, remind the problem of responsibility and fair use of scientific achievements in the sake of progress. We see often that the perceptions of this big issue by scientists and secret services conflicted. History of Physics, specifically in 
connection with biographies of Einstein, Oppenheimer, Scillard, Sakharov and some others recalls that the issue is not just matter of history of Physics but of current actual policy. But, as professor Tilman Sauer recommends, the topic deserves bigger format of discussion beyond this short commentaries. The last point to mention is regret that, if not personal wrangler character and inability of the state system of the Stalin period to mobilize talented intellectuals without repressions into using their abilities into constructive usage of their talents in the interests of their countries instead of destructive opposing to political system, Dmitry Ivanenko might enjoy his scientific productivity including a few of them which were of Nobel level.

Professor D. Ivanenko died in Moscow on December 30, 1994, buried at Kuntsevo Cemetery, Moscow.

\section{Photos with D. D. Ivanenko}

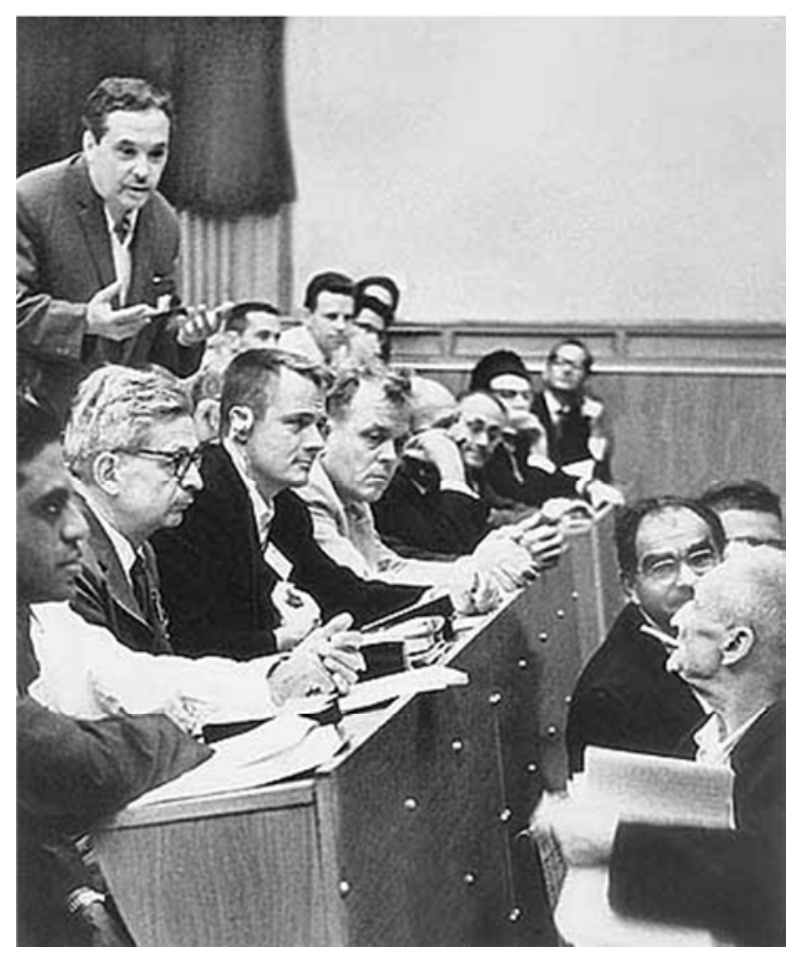

Figure 2. D. D. Ivanenko and I. E. Tamm dispute on the conference in Dubna in 1964. V. Weisskopf is on the right of the Tamm. R. Peierls is the second from the left in the next row. http://vivovoco.astronet.ru/.

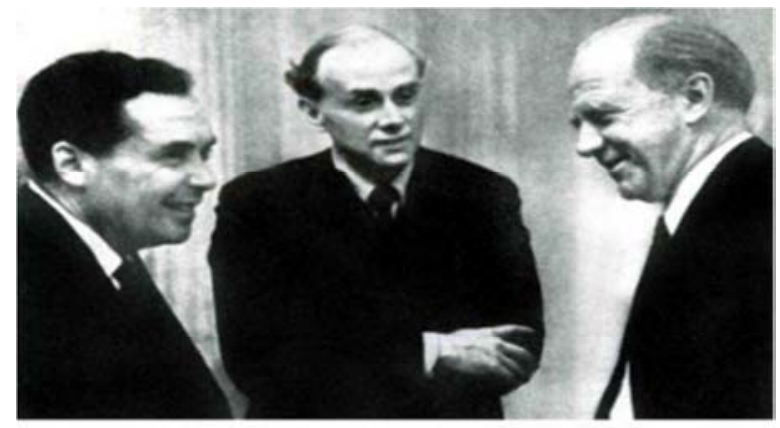

Figure 3. D. Ivanenko, P. A. M. Dirac and W. Heisenberg (Berlin, 1958).

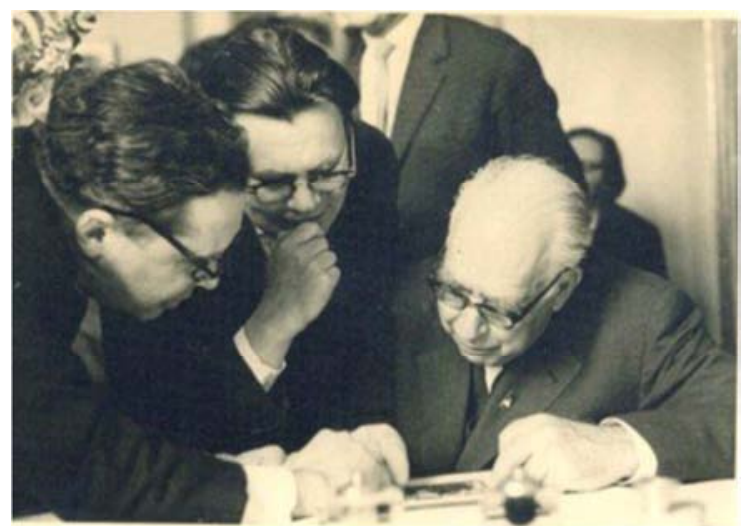

Figure 4. D. Ivanenko and N. Bohr (on the right) in Ivanenko's office in $\operatorname{MSU}(1961)$.

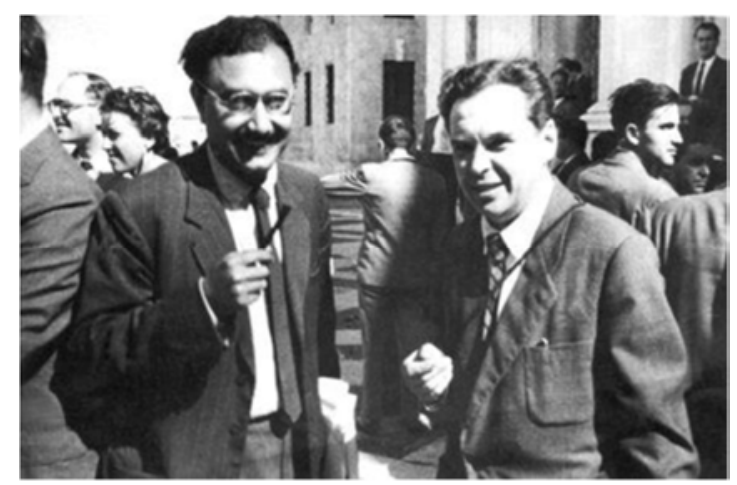

Figure 5. A. Salam and D. Ivanenko.

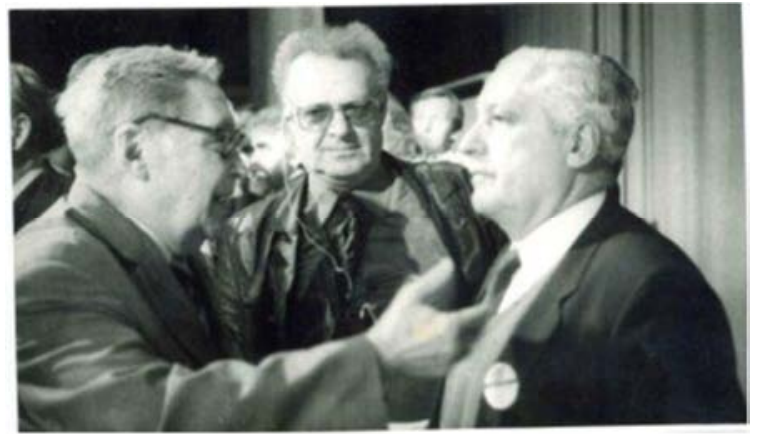

Figure 6. D. Ivanenko and I. Prigogine (on the right) (1981).

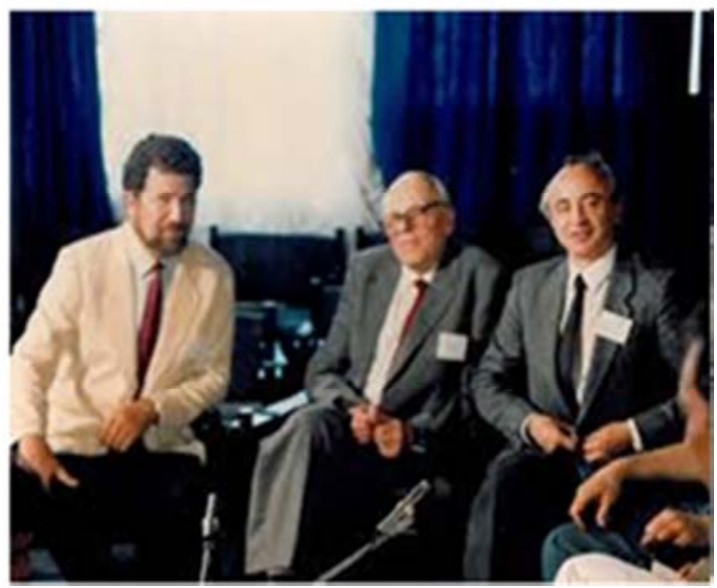

Figure 7. Remo Ruffini, Andrey Sakharov, Igor Novikov. Source: [18] 


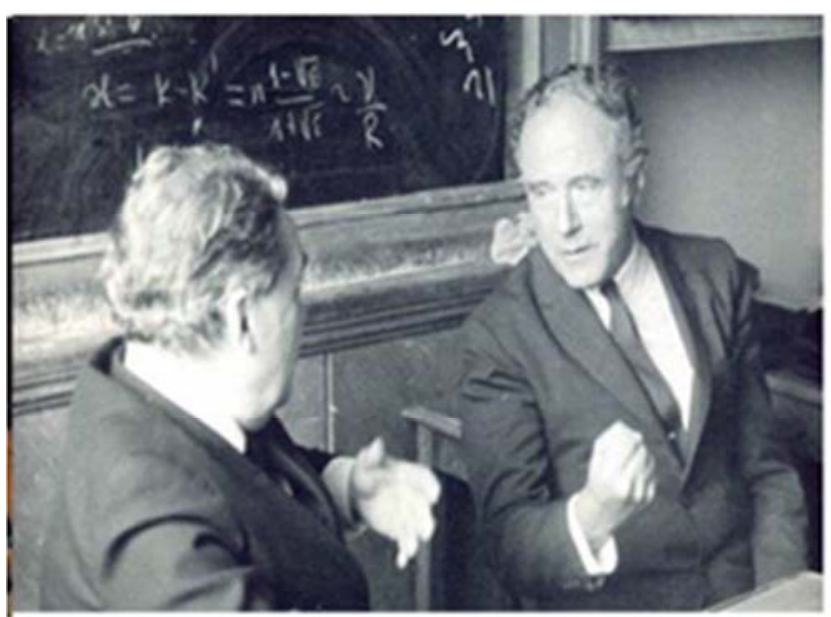

Figure 8. D. Ivanenko and J. Wheeler (1971).

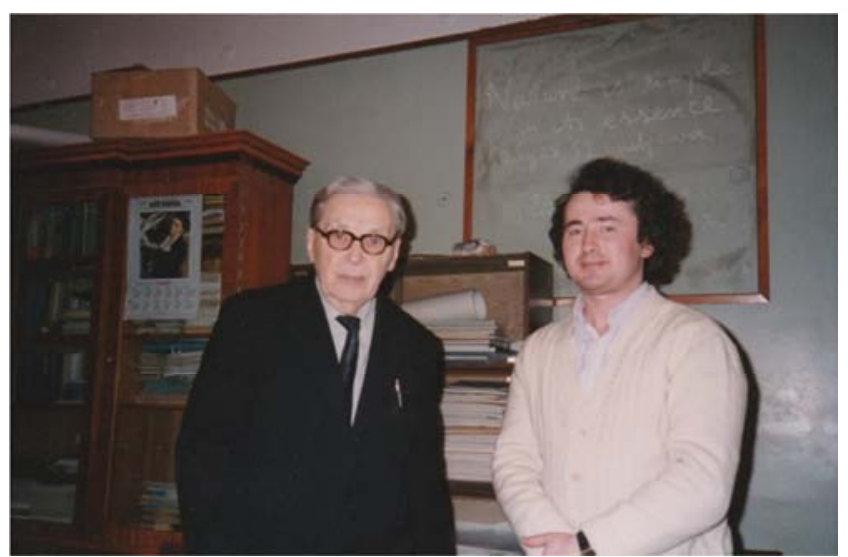

Figure 9. D. Ivananko and the author (1985).

This photo - courtesy of Paul Forman, curator at the Smithsonian Institution, Washington DC, USA - after discussion of the author's thesis concluding, that cosmological term is phenomenological description of the factors such as vorticity and torsion, as well as local rotations and perturbations, and other non-homogenous non-isotropic localities get lost in building symmetrical cosmological models (later formulation see [3-5]). Professor Ivanenko reacted quite emotionally, unexpectedly, saying "please, when I die tell them all that I was long-standing supporter of cosmological term and, therefore, I do not mind if it would be engraved on my tomb "He supported cosmological term"". Hereby, I am delivering his message.

The author was a participant of the seminars held by professor D. Ivanenko, and grateful to the scientific advisor professor V. N. Ponomarev for opportunity to learn from himself, from academician Ya. B. Zeldovich [19] at the Astronomical Sternberg Institute of the Moscow State University named after M. V. Lomonosov and meembers of professor D. D. Ivanenko's seminar. I appreciate my contacts with the group of professor Ivanenko (A. G. Sardanashvili, P. I. Pronon, Yu. N. Obukhov). Condolences with A.G. Sardanashvilis passing away in the 1st of September, while this manuscript was processed in PG Editorial. He was great theoretician, closest pupil-colleague and mean biographer to
Professor D. D. Ivanenko. Sources to photos, if otherwise are not shown, are referenced to his archive.

\section{Concluding Proposal}

We are witnesses of the extremely dramatic productive and counterproductive interaction between scientists and power in different countries at the key episodes of the history. Therefore author calls colleagues into joint international project "Pedagogic and Political Legacies of Great Physicists". UNESCO Chairs Network (UNITWIN) "International Institute for Science and Education Advancement through Disseminating Best Practices" would be the best appropriate format for this initiative (preliminary version). Information wars will be prevented as well as nuclear ones.

\section{Acknowledgement}

This publication is supplementary product of the research supposed to be funded from the Government of Russian Federation, Grant № 11. G34.31.0079.

\section{References}

[1] G. Sardanashvily, "Dmitri Ivanenko - Soviet Physics Superstar: Unpublished Memoirs " (URSS, Moscow, 2010) (in Russian). www.g-sardanashvily.ru/.

[2] https://sites.google.com/site/sardanashvily/. "Gravitation" and "Gauge Gravitation Theory".

[3] Nurgaliev I. S. «Singularities Are Averted by Vortices». Gravitation and Cosmology, 2010, Vol. 16, No. 4, pp. 313-315.

[4] http://www.sinp.msu.ru/en/node/19692.

[5] Nurgaliev I. S "Physics is above All (Yakov Borisovich Zeldovich as Pedagogue - thoughts in his $100^{\text {th }}$ Jubilee". Space, Time and Fundamental Interactions, 2014, vol. 1, pp. 70-76. In Russian.

[6] Nurgaliev I. S. «Cosmology without Prejudice»., Time and Fundamental Interactions, 2014, vol. pp.54-58. (http://www.stfi.ru/journal/STFI_2014_04/nurgaliev.pdf). In English.

[7] Nurgaliev I. S. Physics of the 20th century, global risk and safety, History of Physics in Europe in the 19th and 20th Centuries, Proceedings of $1^{\text {st }}$ Europhysics Conference of European Physical Society, Como, Italy, 1992.

[8] Nurgaliev I. S. Physics and conversion, Proceedings of Soviet Philosophic Conference. Kazan, p. 66 70, 1990.

[9] Nurgaliev I. S. The physical nature of modern arms puts forward the principle of admissible lag instead of parity, Conversion and Physics, Collection of Reports, 1st Soviet Conference of USSR Physical Society, p.19, Kaliningrad, 1991.

[10] Nurgaliev I. S. the Internet from the point of view of education and safety, Works of the $4^{\text {th }}$ International Crimea-97 conference, "Libraries and associations in changing the world: new technologies and new forms of cooperation". Volume 2. P. 387-389. - Sudak, Crimea, 1997. 
[11] Nurgaliev I. S. The Academic Mobility in a Vice of a Spy-mania and Terrorism, In the collection "New info-communication technologies in the social humanities and education: current state, problems, prospects of development". Moscow, Logos, 2003. P. 231-239.

[12] Nurgaliev I. S. What the idea of IHY-2009 consists in?, J. Uspehi fizicheskih nauk. 2009. T. 179. P. 196.

[13] Nurgaliev I. S. The main actions of the International astronomical union within IHY-2009 and sources of more detailed information on them, J. Uspehi fizicheskih nauk. 2009. T.179. P. 200.

[14] Nurgaliev I. S. The international heliophysical year - 2007 under the auspices of the UN, J. Uspehi fizicheskih nauk. 2006. T. 126. P. 566.

[15] Nurgaliev I. S. The Quite Russian. Invasion. International Educator, J. Spring, 1997, p. 42-44.
[16] D. Ivanenko and G. Sardanashvily, The Gauge Treatment of Gravity, Phys. Rep. 94 (1983) 1-45.

[17] Khalatnikov, Isaak M. From the Atomic Bomb to the Landau Institute: Autobiography. Top Non-Secret. Berlin, Heidelberg: Springer Berlin Heidelberg, 2012.

[18] Ruffini, Remo. Moments with Yakov Borisovich Zeldovich. http://arxiv.org/pdf/0911.4825.pdf.

[19] Nurgaliev I. S. Metaphysics in Physics and Mathematics Education in the Twenty-first Century (to the 100th Anniversary of Ya. B. Zeldovich) / / Physics in School. - 2016. - No. 2. - S. 4-13. - ISSN 0130-5522. - Reprinted from: http://www.stfi.ru/documents/STFI_2014_01/STFI_2014_01_ nurgaliev.pdf. 